\title{
Selected Abstracts from the Symposium on the Effects of Chronic Hypoxia on Diseases at High Altitude, August 2008, La Paz, Bolivia
}

\author{
Protection of the Heart Against Ischemic Injury Conferred \\ by Adaptation to Chronic Hypoxia
}

Both epidemiological observations and a majority of experimental studies demonstrated that chronically hypoxic myocardium is more tolerant to acute ischemia/reperfusion injury than control normoxic myocardium. However, the molecular mechanism underlying this long-lasting cardioprotective effect remains unclear. Chronic hypoxia, particularly that of intermittent nature, is associated with increased generation of reactive oxygen species that play an important role in the activation of cell survival pathways. ${ }^{1}$ This observation may imply that the increased cardiac ischemic tolerance afforded by chronic intermittent hypoxia is due to periodic reoxygenation rather than to hypoxia itself. To test this hypothesis, we compared the extent of lethal myocardial injury induced by acute ischemia/ reperfusion (coronary artery occlusion) in adult male Wistar rats adapted to either intermittent hypobaric (high-altitude) hypoxia (IHH, $5500 \mathrm{~m}, 8 \mathrm{~h} \cdot \mathrm{d}^{-1}$ ) or continuous normobaric hypoxia $\left(\mathrm{CNH}, 24 \mathrm{~h} \cdot \mathrm{d}^{-1}\right)$ of the same degree $\left(\mathrm{FIO}_{2}=0.1\right)$. While 15 exposures to IHH were not sufficient to protect the heart, 30 exposures reduced infarct size from $62.5 \% \pm 2.8 \%$ of the area at risk in the normoxic controls to $49.4 \% \pm 3.2 \%$. Continuous normobaric hypoxia provided more efficient protection than IHH: infarct size decreased to $49.0 \% \pm 2.8 \%$ and $41.5 \% \pm 3.0 \%$ after 15 and 30 days of hypoxia, respectively. The protective effect of $\mathrm{CNH}$ was retained in subsequently isolated left ventricular cardiomyocytes subjected to acute anoxia/reoxygenation: cell viability dropped by $8 \%$ compared with $23 \%$ in the normoxic controls, and lactate dehydrogenase release was about half of the control value. Treatment of rats with a selective antagonist of $\beta_{1}$-adrenoceptors metoprolol (50 $\mathrm{mg} \cdot \mathrm{kg}^{-1} \cdot \mathrm{d}^{-1}$ ) during the adaptation period completely prevented the infarct size-limiting effect of $\mathrm{CNH}$. It is concluded that hypoxia per se appears to be responsible for the induction of the protected cardiac phenotype of IHH rats. Catecholamines produced during the adaptation to hypoxia play an essential role in its cardioprotective mechanism, which involves the $\beta_{1}$-adrenoceptor pathway.

Frantisek Kolar, $\mathrm{PhD}$ Jan Neckar, $\mathrm{PhD}$ Gudrun Borchert, PhD Dmitry Stakheev, MD Bohuslav Ostadal, MD, PhD Prague, Czech Republic

(1) Kolar F, Jezkova J, Balkova P, et al. Role of oxidative stress in PKC-d upregulation and cardioprotection induced by chronic intermittent hypoxia. Am J Physiol Heart Circ Physiol. 2007;292:H224-H230.

\section{Hypoxic Adaptation Protects Cardiac Cells Through Autophagy}

Autophagy, an intracellular process of digesting cells' own constituents, has recently been found to be involved in ischemic heart diseases, including heart failure, hypertrophy, and ischemia reperfusion injury. Hypoxic adaptation is known to induce survival signaling in cardiac cells. The present study was undertaken to determine if hypoxic adaptation involved autophagy. To test our hypothesis, H9c2 cardiac myoblast cells were subjected to either 30 minutes of hypoxia followed by reoxygenation for 1 hour (HR) or hypoxic preconditioning, in which the cells were subjected to 3 cyclic episodes of 5 minutes of hypoxia and 5 minutes of reoxygenation, followed by HR. Hypoxia was induced by placing the cultured plates into an air-tight hypoxic chamber placed in a humidified $37^{\circ} \mathrm{C} \mathrm{CO}_{2}$ incubator and passing the mixture of $95 \%$ nitrogen and $5 \%$ $\mathrm{CO}_{2}$. Reoxygenation was induced by placing the cultured plates in a humidified $37^{\circ} \mathrm{C} \mathrm{CO}_{2}$ incubator saturated with air. Hypoxia-reoxygenation injury in $\mathrm{H} 9 \mathrm{c} 2$ cells slightly induced the protein expression of LC3-II, an autophagic marker protein present in the autophagosomal membrane. Also, the protein expression of Bag-1, a multifunctional pro-survival protein that protects cardiac cells from apoptosis, was also induced during hypoxia-reoxygenation. However, hypoxic adaptation prior to hypoxia reoxygenation further induced the protein expression of LC3-II and Bag-1. Our immunofluorescence analysis showed that LC3-II level had been enhanced during hypoxic adaptation. Furthermore, treatment with Bag-1 siRNA attenuated hypoxic adaptation-induced LC3-II and Bag-1 proteins in cardiac myoblast cells. Hypoxic adaptation-induced cardiac cell viability, studied using 3,(4,5-dimethylthiazol-2yl)-2,5-diphenyltetrazolium, was reduced by Bag-1 siRNA treatment. The release of cardiac cell death marker lactate dehydrogenase (LDH), studied using the culture supernatant, was also reduced by hypoxic adaptation, but Bag-1 siRNA treatment abolished the hypoxic adaptation-induced reduction of LDH release. These results indicate that cardiac protection elicited during hypoxic adaptation is mediated at least in part via upregulation of autophagy in association with Bag-1 protein.

\section{Narasimman Gurusamy, $\mathrm{PhD}$ Dipak Das, DK, $\mathrm{PhD}$ Farmington, CT, USA}

\section{Aerobic Capacity in Coca Chewers}

Introduction.--Many Andean people of Peru and Bolivia are used to chewing coca leaves to prevent fatigue during work at 
high altitude. The physiological effects of coca chewing have not been fully explained.

Objective.-The purpose of the study was to investigate the effects of chewing coca leaves on aerobic capacity $\left(\mathrm{Vo}_{2} \mathrm{max}\right)$.

Methods.-The aerobic capacity was measured in 10 coca chewers and 4 non-coca chewers in Cajamarca (2750 m), Peru. The $\mathrm{Vo}_{2} \mathrm{max}$ in submaximal exercise was measured using a cycle ergometer by the Fox test. Coca chewers chewed coca during exercise. The values obtained were calculated with the equation of Fox for men $\left(\mathrm{Vo}_{2} \max =6.3-0.0193 \times \mathrm{FC}\right.$ [cardiac frequency]) and were corrected by the correction factor for the age of Astrand.

Results. - In coca chewers the $\mathrm{Vo}_{2} \max \left(\mathrm{mL} \cdot \mathrm{kg}^{-1} \cdot \mathrm{min}^{-1}\right)$ was $53.02 \pm 3.06$. In non-coca chewers the $\mathrm{Vo}_{2} \max$ $\left(\mathrm{mL} \cdot \mathrm{kg}^{-1} \cdot \mathrm{min}^{-1}\right)$ was $66.59 \pm 6.33$. The difference was not statistically significant.

Conclusion.-The results suggest that there was no difference in the aerobic capacity $\left(\mathrm{Vo}_{2} \mathrm{max}\right)$ in coca chewers and non-coca chewers. The beneficial effects of coca could be metabolic. In all the subjects, aerobic capacity $\left(\mathrm{Vo}_{2} \max \right)$ was classified as either excellent or average.

Elydia Mujica, DSc Jose Aliaga, MSc Nicolas López, MD Lima, Peru Vidayasagar Casikar, PhD Sydney, Australia

\section{Reproductive Hormones in Pregnancy at High Altitude}

Introduction.-There is a similar fertility in high altitude and sea level but studies indicate that there are some differences in pregnancy and childbirth between the inhabitants of high altitude and at sea level. Among these differences are hormone plasma values. Some studies reported that the production of estradiol and progesterone during pregnancy is significantly lower in native inhabitants at high altitude compared with inhabitants at sea level; other studies found lower concentrations of estradiol and estriol but high concentrations of progesterone at high altitude. Others reported that progesterone levels do not change significantly during pregnancy at high altitude compared to sea level, and others found that the urinary excretion of estradiol and estrone are lower at high altitude at the end of pregnancy.

Objective.-The objective is to clarify the controversies found in the literature about reproductive hormone levels in pregnancy and to provide reference values for future studies of the physiological and pathophysiological aspects of pregnancy at high altitude.

Methods.-We measured follicle-stimulating hormone (FSH), luteinizing hormone ( $\mathrm{LH})$, estradiol, and progesterone by radioimmunoassay in pregnant natives of high altitude $(4340 \mathrm{~m})$ and sea level $(150 \mathrm{~m})$ environments who were 3 to 5 months pregnant; 24 women at high altitude and 19 women at sea level were studied.

Results.-The results show that LH and estradiol are significantly lower in pregnant women at high altitude compared with the women at sea level in the third month of pregnancy. In pregnant natives at high altitude, $\mathrm{LH}=6.30 \pm 0.52$ $\mathrm{pg} \cdot \mathrm{mL}^{-1}$; at sea level, $\mathrm{LH}=9.78 \pm 1.52 \mathrm{pg} \cdot \mathrm{mL}^{-1}(P<.05)$. In pregnant natives at high altitude, estradiol $=2662 \pm 351$ $\mathrm{pg} \cdot \mathrm{mL}^{-1}$; at sea level, estradiol $=3851 \pm 434 \mathrm{pg} \cdot \mathrm{mL}^{-1}(P$ $<.05$ ). We did not find significant differences in the other hormones studied.

Conclusion.- There are no differences between progesterone and FSH during pregnancy at high altitude and sea level. At high altitude, the reduction in $\mathrm{PO}_{2}$ affects the production of $\mathrm{LH}$ and estradiol at the beginning of pregnancy.

\section{Elydia Mujica, DSc Manuel Yampufe, MSc Jose Aliaga, MSc Gerardo Ronceros, MSc Lima, Peru}

\section{Does Chewing Coca Leaves Influence Physiology at High Altitude?}

It is well known that Peruvian highlanders are in the habit of chewing coca leaves while working in the mountains. It is speculated that they do not suffer from effects of hypoxia to the same extent as those who do not use coca leaves. We are trying to find out if the coca leaf chewing influences hypothalamus-pituitary-adrenal response to the stress induced by high altitude.

We subjected 10 healthy adult males at Cajamarca to a standardized exercise routine and measured blood pressure, oxygen saturation, pulse, $\mathrm{Vo}_{2} \mathrm{max}$, and cardiac function (Holter monitor). These subjects were made to chew coca leaves during the period of exercise, which lasted for 2 hours and 30 minutes. They were on the exercise cycle for a period of 20 minutes at a time followed by period of rest for 20 minutes. Blood samples were taken at the beginning and at the end of the exercise. Microdialysis samples were collected at intervals of $20 \mathrm{~min}$ utes. Four subjects who did not use coca leaves were used as controls.

We estimated the levels of testosterone and progesterone in the blood (reported separately) and the levels of glutamate, glucose, glycerol, lactate, and pyruvate in subcutaneous tissue using microdialysis probes supplied by Global Scientific.

1. Pyruvate level was elevated, indicating increased glucose utilization. Under hypoxic conditions, glucose utilization is impaired, as indicated in the controls. This suggests that coca users meet the energy requirements at higher levels. This needs to be further revalidated by estimating pyruvate dehydrogenase activity.

2. Increase in glucose levels by coca users is probably facilitated through increased transport of glucose across the intracellular membrane, enhanced by glucose transporters, which are insulin dependent.

3. The lactate levels were higher in coca users. This is due to the increase in pyruvate. This was seen to decrease gradually, as the pyruvate was used at the expense of lactate.

4. Elevated glycerol in coca users was probably due to in- 
creased fat and glucose metabolism. The influence of betaoxidation and aerobic glycolysis needs further study with more data.

5. The coca chewers had lower levels of glutamate compared to controls. Glutamate is the basis for many neurotransmitters and is the product of amino acid metabolism. This is an indication of reduced oxygen utilization.

6. Blood pressure, pulse rate, cardiac function, and $\mathrm{Vo}_{2}$ max did not show any variations between the coca users and the controls.

Coca chewing shows subtle biochemical effects. The problems at high altitude are due to stress. Andean Indians have recognized the benefits of the coca plant and have incorporated it into their culture wisely (unlike the advanced Western cultures). We hope to study this controversial issue further.

Vidyasagar Casikar, MD Elydia M. Mujica Alban, DSc Carol Smith

Franchesca Bartholomew Jose Aliaga Arauco, MSc Lima, Peru

Usefulness of Biochemical Markers in the Evaluation of the Effect of Pranayama During the Management of Chronic and Acute Mountain Sickness

Of late, a large number of lowlanders are going to high-altitude conditions for various reasons. Most of them do suffer from acute mountain sickness. Prevention of mountain sickness is of prime importance, as the treatment of any sick individual requires complicated logistics. It is here that several alternate and holistic approaches have been made by man for a very long time. Several techniques involving regulated breathing or pranayama seem to be of great value. It is only of late that biochemical markers used to quantify mountain sickness have been identified. Understanding of the biochemical markers to evaluate the effect of pranayama in mountain sickness is of great importance. The beneficiary effects of pranayama or regulated breathing are well established in normal healthy individuals and in several cases of sick persons at various altitude conditions. Nidisodhana, which is one of the basic techniques of pranayama, is found to have maximum beneficial effects; it represents a means of conducting a breathing exercise through alternate nostrils with inhalations and exhalations in the ratio of 1:2 in a sitting posture (Vajrasana) with durations of 8 and 16 seconds. When this is practiced for 30 minutes it is found to have produced an increased concentration and self-awareness. This simple breathing exercise is found to reduce body fat. Hence, all factors of the lipid profile become the indicators to assess the effects of regulated breathing. This is more so the case with lowlanders who are exposed to high-altitude conditions. In high-altitude conditions, where the availability of oxygen is less and where much effort is needed on the part of the physiological part of the system of pranayama. Reactive oxygen species are known to aggravate disease progression. To counteract their harmful effects, the body produces various antioxidant enzymes (viz, superoxide dismutase, glutathione re- ductase, etc). Literature reviews revealed that exercises help to enhance antioxidant enzyme systems; hence, yogic exercises such as pranayama may be useful to combat various diseases. Apart from the lipid profile, even blood glucose seems to be one of the earliest biochemical markers to quantify the effects of pranayama. Several findings indicate that pranayama exercises have enhanced the antioxidant defense mechanism in diabetics by reducing oxidative stress. With the many other beneficial effects of pranayama, one can notice significant changes in the following: increased lung capacity, improved immune system, recharged nervous system, reduced stress, and improved memory.

Though biochemical markers for many of the above indicated aspects are not clearly understood, they can be studied through the various hormones and neurotransmitters that play significant roles. It is proposed to use this simple pranayama technique to simulate various physiological processes and to keep tracking the levels of various biochemical indicators or markers to prevent the deleterious effects of mountain sickness.

Thuppil Venkatesh, MD Bangalore, India

\section{The Low and High Altitude Hypoxia Faces}

Hypoxia at low and high altitude or at sea level is a widely spreading state. On one hand, some use the term "hypoxia" when there are external or internal signals about the lack of oxygen supply to the organism, organs, or cells, and often the term is used without direct proof. It is well known that acute or chronic hypoxia may cause mountain sickness, tissue damage, insults, infarcts, and different pathologic states. On the other hand, periodic (intermittent) hypoxia may act as a training factor, enlarge the mitochondria pool of cells, stimulate cell proliferation in organs, and enforce the human's work and sports capacity. These 2 opposite hypoxia actions arise on the basis of our inability to exactly determine the degree of hypoxia and length of exposure for different people, animals, and other biological beings as a result of individual and species sensibility to low oxygen partial pressure and its duration.

Some years ago we proposed to distinguish the sanogenetic and pathogenetic hypoxia types. For the healthy man, sanogenic hypoxia begins from 1000 to $3000 \mathrm{~m}$ above sea level (m.a.s.l.) or at an equivalent dose in gas mixtures. The highaltitude and long-term stay may cause pathogenic effects.

For cardiac or pulmonary illness, the sanogenic level begins at $500 \mathrm{~m}$ and finishes at about 1500 to $2000 \mathrm{~m}$.a.s.l. Slow ascent with "step adaptation" after N. Sirotinin, because the time factor plays a very important role, may be the adequate procedure.

Most countries have no mountains and thus need instrumental-type orotherapy-hemipermeable polymer films that can separate oxygen and nitrogen molecules from ambient air. Such an apparatus is produced in the Ukraine using Japanese polymer membranes. We named it "Borey" and it is used to supply special clinics and Black Sea resorts. Artificial mountain air from "Borey" consists of $10 \%$ to $12 \% \mathrm{O}_{2}$ in nitrogen 
and light electronegative airions. It was shown that intermittent hypoxia treatments stop development of bronchial asthma attacks, vegetative dystonia symptoms, and headache as well as shortening chronic bronchitis duration. At the same time, healthy people became more resistant to chronic fatigue symptoms and acquired a higher resistance to stress.

\section{Vadim A. Berezovskii, MD Kiev, Ukraine}

\section{Does Breath Holding Time Increase With Adaptation to High Altitude?}

Breath holding time is restricted by various physiological mechanisms that prevent inadequate gas exchange. At sea level, breath holding time is limited by the accumulation of carbon dioxide, which stimulates ventilation, and breath holding is terminated. This will also occur at high altitude; however, in this hypoxic environment oxygen levels seem likely to play an influential role. Furthermore, the normal $\mathrm{PaCO}_{2}$ at $3510 \mathrm{~m}$ in the city of La Paz, Bolivia, is $30 \mathrm{~mm} \mathrm{Hg}, 10 \mathrm{~mm} \mathrm{Hg}$ less than the normal value at sea level. Therefore, as the body adapts to altitude, breath holding time may change. We hypothesized that breath holding time would increase with acclimatization. To test this hypothesis, breath holding studies were performed in an unacclimatized individual over a 2-week period following the individual's arrival in La Paz. A number of parameters were recorded, including oxygen saturation, heart rate, end-tidal $\mathrm{CO}_{2}$, and breath holding time. The breath holding time was recorded after a monitored period of normal breathing during 2 minutes. No significant difference was noted in breath holding times during the first 2 weeks of adaptation to high altitude. The respiratory center's breath holding time is not influenced by a normal acute adaptation exposure to a fixed high altitude, and it is linked to the immediate adaptation of the organism to a lower $\mathrm{PaCO}_{2}$ at high altitude.

Luis Zubieta-Calleja, MD

Gustavo Zubieta-Castillo, MD

Joanna Kingston, MD

Gustavo Zubieta-Calleja, MD

La Paz, Bolivia

\section{The Ventilatory Hyperoxic Shunt Test at 3510 m}

We use a new method to test pulmonary shunt by hyperoxia at high altitude. The subject is tested while first breathing am- bient air in the seated position. Using a face mask, ventilation, $\mathrm{ETO}_{2}, \mathrm{ETCO}_{2}$, electrocardiography, pulse oximetry, and blood pressure are measured. The breath-by-breath system is online and via analog digital converters registered in a computer. An arterial blood sample is taken from the radial artery. Then the subject breathes $89 \%$ oxygen during 6 minutes while the data collection continues. A $100 \% \mathrm{O}_{2}$ would be better, but these were not available. This technique allows for precise measurements that provide a clear picture of cardiorespiratory parameters. Furthermore, it allows the possibility of observing when there is an equilibration of the $\mathrm{PIO}_{2}$ as it is observed on a breath-by-breath basis in the same wave in which the $\mathrm{ETO}_{2}$ is observed. The average $\mathrm{PIO}_{2}$ was $421.7 \mathrm{~mm} \mathrm{Hg}$. For $3510 \mathrm{~m}$, the altitude of our laboratory, the test was performed on 14 normal subjects (mean $\pm \mathrm{SD}$ ) using t-students' reports (see Table).

This test permits the operator to observe when the $\mathrm{PIO}_{2}$ reaches the maximum level, thereby making the precise observation of shunt, regardless of uneven ventilation that can delay such equilibration of inspired gases. The intra-pulmonary shunts are essential for evaluation of patients with chronic mountain sickness at high altitude.

\section{Gustavo Zubieta-Castillo, MD \\ Luis Zubieta-Calleja, MD \\ Joanna Kingston, MD \\ Gustavo Zubieta-Calleja, MD \\ La Paz, Bolivia}

\section{The Adaptation of Highlanders to Sea Level}

If high-altitude residents go to sea level, their organism is facing a "relative hyperoxia" and, hence, an aggression of an abnormal environment (disregarding the other factors, such as temperature and humidity, food, lodging, and so on). The aggressor happens to be oxygen, a combustion accelerator.

In 1975, 6 young men born and living in La Paz $(3600 \mathrm{~m})$ were taken to Puerto Villarroel (500 m above sea level), a small tropical town in Chapare, Bolivia. Ventilation was measured using a Wright Respirometer the day after arrival, and the average decreased from $13128 \mathrm{~mL} \cdot \mathrm{min}^{-1}$ (BTPS), the resting value in $\mathrm{La} \mathrm{Paz}$, to $2300 \mathrm{~mL} \cdot \mathrm{min}^{-1}$ (BTPS). Soldier residents in the same location had an average ventilation of $4063 \mathrm{~mL} \cdot \mathrm{min}^{-1}$ (BTPS).

The adaptation mechanism acquired over long-term high-

Table. Pulse oximetry, ventilation, and arterial blood gases in the modified pulmonary function test*

\begin{tabular}{|c|c|c|c|c|c|c|c|}
\hline & $\begin{array}{c}\mathrm{SpO}_{2} \\
(\%)\end{array}$ & $V E B T P S$ & $\begin{array}{c}\mathrm{ETCO}_{2} \\
(\mathrm{~mm} \mathrm{Hg})\end{array}$ & $\begin{array}{c}\mathrm{ETO}_{2} \\
(\mathrm{~mm} \mathrm{Hg})\end{array}$ & $\begin{array}{c}\mathrm{PaO}_{2} \\
(\mathrm{~mm} \mathrm{Hg})\end{array}$ & $\begin{array}{c}\mathrm{PaCO}_{2} \\
(\mathrm{~mm} \mathrm{Hg})\end{array}$ & $p H$ \\
\hline Ambient air & $91.56 \pm 1.45$ & $9.19 \pm 2.3$ & $28.05 \pm 1.9$ & $66.42 \pm 4.05$ & $59.3 \pm 5.92$ & $27.7 \pm 3.72$ & $7.43 \pm 0.03$ \\
\hline $89 \% \mathrm{O}_{2}$ & $98.15 \pm 0.73$ & $8.26 \pm 2.06$ & $26.17 \pm 2.2$ & $337.59 \pm 14.7$ & $237 \pm 25.8$ & $27.25 \pm 3.6$ & $7.46 \pm 0.06$ \\
\hline$P$ & $<.0001$ & NS & $<.0001$ & $<.0001$ & $<.0001$ & NS & NS \\
\hline
\end{tabular}

$* \mathrm{SpO}_{2}=$ Oxyhemoglobin saturation measured by pulse oximetry. VE(BTPS) = Ventilation in one minute (Body Temperature Pressure Saturated). $\mathrm{ETCO}_{2}=$ End-tidal carbon dioxide. $\mathrm{ETO}_{2}=$ End-tidal oxygen. $\mathrm{PaO}_{2}=$ arterial oxygen tension. $\mathrm{PaCO}_{2}=$ arterial carbon dioxide tension. $\mathrm{pH}=$ arterial blood $\mathrm{pH}$. NS $=$ not significant. 
altitude residence is essentially the high hematocrit. The highaltitude normal adaptive erythrocythemia would, in the presence of excess oxygen, increase the oxygen content of blood and produce hyperoxemia. Resultant hypoventilation in turn diminishes water loss and gives rise to water retention and is particularly evident to people suffering from inadequate adaptation to sea level. High-altitude residents who go to sea level often feel that their shoes are tight. This is due to edema during the first days in some and persists several days in others, particularly if kidney function is not $100 \%$ effective. Furthermore, some feel drowsy, tired, and sleepy for a few days, and others complain of headaches.

On immediate descent of chronic mountain sickness patients, there is bright red color in the cheeks, simulating the face of the Pink Puffer, but without the shortness of breath. The full oxygen saturation of blood with a very high hematocrit is the explanation. As the hematocrit decreases, the color of the skin changes to become more pale, along with the conjunctivae and the mucosa.

The time for the hematocrit to reduce to normal levels is around 25 days going from $3510 \mathrm{~m}$ to sea level. The highaltitude adaptation formula previously described,

$$
\text { Adaptation }=\frac{\text { Time }}{\text { Altitude }}
$$

also applies in a reverse way. Upon ascent to high altitude it has been determined that the multiplication factor in order to calculate the hematocrit adaptation time is 11.4 days per $1 \mathrm{~km}$ of altitude. In order to adapt to $3510 \mathrm{~m}$ it is necessary to multiply 3.5 by 11.4 , giving a time lapse of 40 days. In a reverse phenomenon, upon descent, replacement of red blood cells stops, and there is a gradual linear decrease of the hematocrit.

Gustavo Zubieta-Calleja, MD Poul-Erik Paulev, MD, Dr Med Sci Gustavo Zubieta-Castillo, MD La Paz, Bolivia

The P-50 of the Hemoglobin Oxygen Dissociation Curve of the Chipayas: A 2000 Year High Altitude Dweller Bolivian Ethnic Group

The Chipaya are an indigenous Andean culture dating back more than 4500 years who, in the face of aggression over water and arable lands from competing ethnic groups like the Inca or Aymara, were forced to retreat to an isolated area on the Bolivian altiplano. They situated themselves in Bolivia's southwest, beside the Salar de Coipasa (4100 m), on barren land, where they subsisted by fishing and farming. Their isolation persisted until very recently, when they began to travel to Chile and other parts of Bolivia for work, which has consequently affected their culture in radical ways. They have been the subject of study for many world-renowned anthropologists who believed they were on the verge of extinction. Quite to the contrary, the population has increased, and they have adapted their living conditions to those of other altiplano towns. Their typical cone-shaped homes have given way to traditional Altiplano Calamina houses, and today, in addition to bicycles, they travel long distances in modern vehicles.

In 2003 the authors collected blood samples from the antecubital veins of 11 male Chipaya, not a simple task, as they are very suspicious of strangers and their cultural histories include stories of vampire-like creatures. The sample included a broad range of ages: subjects ranged from 15 to 78 years of age, with an average age of $44.47 \pm 22.47$ years. The mean hematocrit was $51.5 \% \pm 7.07 \%$, which is not significantly different from the normal high-altitude population of La Paz. Two subjects were found to suffer from CMS, and this was previously reported at the Seventh World Congress on Mountain Medicine in Tibet. The blood samples were analyzed using the Ryan Neville Yeast Cells oxygen dissociation curve technique, modified with a computerized system. The results demonstrate that the oxygen dissociation curves are normal in shape, and the P-50 was found to be $23.13 \pm 1.54$ (SD), clearly revealing a left-shifted curve in this ethnic group relative to normal sea level values.

$$
\begin{array}{r}
\text { Gustavo Zubieta-Castillo, MD } \\
\text { Michael Moretti, BS } \\
\text { Gustavo Zubieta-Calleja, MD } \\
\text { La Paz, Bolivia }
\end{array}
$$

\section{Cardio-Pulmonary Response and Lung Interstitial Fluid Dynamics in Chronic Hypoxia}

Acute hypoxia exposure $\left(10 \% \mathrm{O}_{2}\right)$ causes an increase in cardiac output and in alveolar ventilation, both effects aiming to defend the supply of oxygen to the tissues. Acute hypoxia also leads to an increase in pulmonary artery pressure, mostly reflecting an increase in precapillary arteriolar resistance. Hypoxia also causes capillary recruitment; therefore, this vascular response may be interpreted as a mechanism to offset the increase in microvascular filtration due to the hypoxia-induced increase in microvascular permeability. In previous work ${ }^{1}$ we found that pulmonary cells, in particular endothelial cells, act as sensors of the mechanical situation in the interstitial space. In fact, we found that after acute hypoxia exposure, the signaling-transduction response was evoked in vivo by an increase in pulmonary interstitial pressure (Pip) during development of interstitial edema. ${ }^{2,3}$ We now examine how pulmonary interstitial fluid dynamics evolve in Wistar adult rats exposed to chronic hypoxia ( $10 \% \mathrm{O}_{2}$ for up to 3 months). Pulmonary interstitial pressure was measured by micropuncture technique and was found to average $\sim 15 \mathrm{~cm} \mathrm{H}_{2} \mathrm{O}$ in chronic hypoxic rats, compared to a control value of $\sim-7 \mathrm{~cm}$ $\mathrm{H}_{2} \mathrm{O}$ in matched animals. The increase in Pip stems from a sustained condition of interstitial lung edema in chronic hypoxia, suggesting a permanent activation of signaling-transduction response by lung cells to induce interstitial matrix turnover. We hypothesize that in chronic hypoxia, capillary compression by tissue forces may cause an increase in microvascular resistance that may contribute to the steady increase in pulmonary artery pressure.

Giuseppe Miserocchi, MD Ilaria Rivolta, $\mathrm{BD}, \mathrm{PhD}$ Monza, Italy 
(1) Daffara R, Botto L, Beretta E, et al. Endothelial cells as early sensors of pulmonary interstitial edema. J Appl Physiol. 2004;97:1575-1583.

(2) Botto L, Beretta E, Daffara R, Miserocchi G, Palestini P. Biochemical and morphological changes in endothelial cells in response to hypoxic interstitial edema. Respir Res. 2006;13:7.

(3) Miserocchi G, Passi A, Negrini D, Del Fabbro M, De Luca G. Pulmonary interstitial pressure and tissue matrix structure in acute hypoxia. Am J Physiol Lung Cell Mol Physiol. 2001;280:L881-L887.

\section{Acetazolamide and Hypoxic Ventilatory Response: Are the} Carotid Bodies Inhibited?

Acetazolamide (AZ) is well known for its beneficial effect at high altitude to prevent and treat acute mountain sickness (AMS). It induces a metabolic acidosis, a rise in ventilation and arterial $\mathrm{PO}_{2}$, and a fall in arterial $\mathrm{PCO}_{2}$ of about $1 \mathrm{kPa}$. One of the potential mechanisms by which the agent ameliorates AMS symptoms and sleep quality is by improving tissue oxygenation. However, decreasing chemoreflex gain of the carotid bodies has also been suggested as a potential mechanism. In a double-blind placebo-controlled randomized study in 9 healthy subjects we investigated the effect of AZ $(250 \mathrm{mg}$ every 8 hours during 3 days) on the $\mathrm{O}_{2}-\mathrm{CO}_{2}$ interaction in the carotid bodies (ie, on the ability of $\mathrm{CO}_{2}$ to increase the isocapnic acute hypoxic ventilatory response [AHR], defined as delta ventilation/delta $\log \mathrm{PaO}_{2}$ ). The AHR was measured at 3 levels of constant end-tidal $\mathrm{PCO}_{2}$ after placebo and $\mathrm{AZ}$. Then, the AHR was plotted as a function of arterial $\left[\mathrm{H}^{+}\right]$, measured from arterial blood samples. Acetazolamide induced a metabolic acidosis and increased ventilation and shifted the AHR$\left[\mathrm{H}^{+}\right]$response line to higher levels of arterial $\left[\mathrm{H}^{+}\right]$without a change in slope, indicating no effect on the $\mathrm{O}_{2}-\mathrm{CO}_{2}$ interaction. At approximately resting $\mathrm{PCO}_{2}$ levels the AHR was approximately the same under both conditions, despite the considerable acidosis after AZ. Both after placebo and AZ, infusion of bicarbonate induced a parallel shift of the AHR- $\left[\mathrm{H}^{+}\right]$response lines to the left. The results of this study indicate that at the dose applied AZ has no inhibitory effect on the carotid bodies and that the arterial $\mathrm{H}^{+}$concentration as such has no predictive value with regard to the magnitude of the AHR. In metabolic acidosis the AHR does not increase because the $\mathrm{PCO}_{2}$ falls, indicating that the intracellular $\mathrm{pH}$ is an important parameter determining the magnitude of the AHR.

Luc J. Teppema, $\mathrm{PhD}$

Eveline van Dorp Frank de Wit

Jack van Kleef Albert Dahan

Leiden, The Netherlands

The Oxyhemoglobin Dissociation Curve In Vivo. Part I: Gaining Control of $\mathrm{PO}_{2}$ and $\mathrm{PCO}_{2}$ Independently of Each Other and Independently of Minute Ventilation

Hyperventilation on acute exposure to altitude increases $\mathrm{SpO}_{2}$ by increasing $\mathrm{PAO}_{2}$ and by shifting the oxyhemoglobin dis- sociation curve to the left. The hypocapnia also reduces cerebral blood flow. It is possible to maintain isocapnia, but to determine if it is worth doing, one must measure the relative contribution of each mechanism to $\mathrm{SpO}_{2}$. There are 5 basic principles that can be applied to prospectively set the alveolar ventilation for $\mathrm{CO}_{2}$ and $\mathrm{O}_{2}$ independently of each other with a simple breathing circuit. I will illustrate the method by using these principles to design the methodology of the experiment to answer the following question: How does hyperventilation contribute to an increase in $\mathrm{SpO}_{2}$ ?

\section{Joseph A. Fisher, MD, FRCP(C) Toronto, Canada}

The Oxyhemoglobin Dissociation Curve In Vivo. Part II: A Field Test of Acclimatization and Adaptation to Altitude?

A left shift of oxyhemoglobin dissociation curve (ODC) is the major functional adaptive strategy to hypoxia in animals living at altitude. The increased $\mathrm{O}_{2}$ affinity of haemoglobin functions as a "sink" for $\mathrm{O}_{2}$ diffusing into the plasma and thus maintains the diffusion gradient. In humans there is no change in the position of ODC, but with acute exposure to altitude, hyperventilation reduces the $\mathrm{pH}$ and induces a temporary shift of the ODC as a form of acute acclimatization. Our data showed that at $4700 \mathrm{~m}$ the contribution of hyperventilation to the increase in $\mathrm{SpO}_{2}$ is primarily due to an increased alveolar $\mathrm{PO}_{2}$ and to a lesser-but still significant—extent, the left shift of the ODC. However, at greater altitude, the relative contribution of these factors will likely be reversed.

Joseph A. Fisher, MD, FRCP(C) Toronto, Canada

Effect of Interval Normobaric Hypoxic Training (IHT) on the Mitral Annular Motion in Hypertensive Patients

Background.-Hypertension is one of several risk factors of cardiovascular diseases, and it is associated with left ventricular (LV) systolic and diastolic dysfunction. Subendocardial function is affected early in hypertensive heart disease and by altering LV long axis motion. It is known that longitudinal LV function is a major determinant of global ventricular function and is probably more sensitive than radial function in the detection of disease. Longitudinal LV function is depressed in hypertensive patients (HP). Tissue doppler imaging of the mitral annulus (ma) allows the study of longitudinal LV function. We tried to determine the influence of interval normobaric hypoxic training (INHT) on the dynamics of the mitral annular motion in systole and diastole in HP.

Methods. - The course of INHT $(23.5 \pm 1.0$ sessions $)$ was presented to 16 patients (male/female, $5 / 11$; mean age, $49.3 \pm$ 1.1 years). They underwent standard 2-dimensional echo studies. Diastolic function was assessed by peak early and late mitral doppler flow velocity (E-TMF, A-TMF) and pulmonary venous systolic/diastolic velocity ratio (S/D-PVF). Tissue doppler images (TD) were analyzed in off-line mode (Echopac 6.3.3 software) from apical position 4- and 2-chamber views using a Vingmed System FiVe (GE). Mitral annular systolic 
(S) and early diastolic (E) velocities were extracted from colorcoded images in 4 sites: septal (sep), lateral (lat), inferior (inf), and anterior (ant).

Results.-Parameters pre- and post hypoxic training are presented in the table. It is known that velocities $\mathrm{Sa}$ and $\mathrm{Ea}$ are decreased in hyertensive patients. Moreover, cross-correlation between E and Ea gets lost. Having performed INHT we noticed that the velocity Sa increases in the three sites and velocity Ea increases at all sites of the mitral annulus. The increased Sa post INHT is associated with improvement of the left atrial reservoir function. In is known that ratio S/D shows relation of left atrial reservoir to left artrial conduit function. The ratio S/D is increased post INHT. Dynamics of cross-correlation between $\mathrm{E}$ and Ea post INHT has shown increase of correlation with each point: E lateral $(r=0.06$ pre and 0.40 post INHT), E septal ( $r=-0.07$ pre and $r=0.33$ post INHT); E inferior ( $r=-0.04$ pre and $r=0.36$ post INHT); E anterior $(r=0.03$ pre and $r=0.34$ post INHT). We suggest recombination of cross-correlation between $\mathrm{E}$ and Ea post INHT reflects improvement in LV relaxation.

Conclusion.- This study shows that 1) longitudinal function at hypertensive patients was improved post-INHT; and 2) Data may be useful in future studies for evaluation of the efficacy treatment and/or high-altitude training.

\section{Klara Drugova Inna Gofman Magnitogorsk, Russia}

\section{Molecular Mechanisms of Acclimatization to High Altitude Hypoxia: Role of Hypoxia Mimetic (Cocl2)}

Adverse environmental conditions such as extreme cold and hypoxia in particular, prevailing at high-altitude locations, are the root cause of many mountain maladies (viz, acute mountain sickness, high-altitude cerebral edema, and high-altitude pulmonary edema) that develop in unacclimatized persons shortly after ascent to high altitude. This study reports the molecular mechanisms of action by cobalt, a hypoxia mimetic, in facilitating acclimatization to hypobaric hypoxia using male Sprague-Dawley rats as a model system. Hypoxic gasping time and survival time were determined as a measure to assess the degree of tolerance of animals to hypobaric hypoxia by exposing the animals to an altitude of $10668 \mathrm{~m}$. Oral administration of cobalt chloride $\left(12.5 \mathrm{mg} \mathrm{Co} \cdot \mathrm{kg}\right.$ body weight ${ }^{-1}$ for 7 days) increased gasping time and hypoxic survival time by 3 to 4 times as compared to the control animals. This could be attributed to an increased expression and DNA binding activity of hypoxia inducible transcriptional factor and its regulated genes (viz erythropoietin, vascular endothelial growth factor [VEGF], glucose transporter-1, and nitric oxide synthase levels in rat brain). This probably leads to improved oxygenation, glucose transport, and maintenance of vascular tone, respectively, under conditions of limited oxygen availability. Exposure of animals to hypobaric hypoxia also resulted in a significant increase in vascular leakage in the brain of rats (an indicator of cerebral edema) as well as reactive oxygen species (ROS), nitric oxide, and VEGF levels. There was a marked increase in NFkB DNA binding activity and levels of proinflammatory cytokines such as monocyte chemoattractant protein, interferon-g, interleukin- 1 , and tumor necrosis factor- $\alpha$ and cell adhesion molecules such as VCAM-1 and P-selectin. Chemical preconditioning by cobalt for 7 days significantly attenuated cerebral vascular leakage, ROS generation, and the expression of inflammatory mediators induced by hypoxia. The lower levels of NFkB observed in the brain of cobaltadministered animals might be due to higher levels of antioxidant and anti-inflammatory proteins (hemeoxygenase- 1 and metallothionein). The findings of the present study provide a basis for the possible use of cobalt for prevention of hypoxiainduced oxidative stress, vascular leakage, and facilitation of acclimatization to hypoxia and other conditions involving oxygen deprivation.

\section{Anju Bansal \\ K. Srivastava \\ S. Suryakumari \\ M. Sairam \\ G. Ilavazhagan \\ Dehli, India}

\section{Interval Hypoxic Trainings as a Method for Rehabilitation of Children and Adolescents with Arterial Hypertension}

The impact of unfavorable environmental factors and increased information overloading on the children's organism leads to the tension of stress-limiting systems and early development of arterial hypertension ( $\mathrm{AH})$ as a classic model of psychosomatic disorder. Mild sparing physiotherapeutic therapies that influence the main pathogenetic chains are preferable in the initial stages of the pathology. These factors are interval hypoxic training or normobaric hypoxic therapy (NH). We modernized the method of conducting $\mathrm{NH}$ in children and adolescents in our clinic, which allowed us to extend the spectrum of the application of the given physical factor and to avoid negative vegetative effects on a child's organism. We also carried out a prospective randomized cohort study of 91 adolescents with $\mathrm{AH}$ (aged 12 to 17 years) who had a course of $\mathrm{NH}$, according to the modernized method, as a nonpharmacological correction. The efficiency criteria were the parameters of arterial pressure, sympathoadrenal reactivity, level of anxiety, and the state of the vascular tonus in the basin of medial cerebral artery. The data obtained were compared with the data from the control group (75 adolescents) analogized in terms of sex and age. The data were standardized; informative symptoms were marked out. We received valid distinctions in the group of patients who had normobaric hypoxytherapy as standardization of the efficiency criteria. At the same time, no valid distinctions were obtained in the control group. Thus, interval hypoxic trainings are an effective component in the complex rehabilitation of children and adolescents with $\mathrm{AH}$.

Vladimir Dolgikh, Prof Lubov Kolesnikova, Prof

Lubov Rychkova, Prof Anastasia Vlasenko, $\mathrm{PhD}$ Olga Bugun, $\mathrm{PhD}$ Irkutsk, Russia 
Global Gene Expression Profiling in Rat Brain Exposed to Hypobaric Hypoxia and the Effect of Cobalt Preconditioning

Ascent to high altitude by millions of people annually results in the risk of altitude sickness worldwide, including acute mountain sickness, high-altitude pulmonary edema, and highaltitude cerebral edema. High-altitude cerebral edema is the most severe form of altitude sickness, and if no intervention is implemented, it may even lead to death. Despite several studies, complete understanding of underlying mechanisms still remains elusive. The present study was designed to understand the effects of hypobaric hypoxia and chemical preconditioning by hypoxia mimetic cobalt $(12.5 \mathrm{mg} \mathrm{Co} \cdot \mathrm{kg}$ body weight $^{-1}, 7$ days, oral) on rat (male, Sprague-Dawley) brain by microarray. Rats were subjected to an altitude of $9142 \mathrm{~m}$ $\left(6 \% \mathrm{O}_{2}\right)$, followed by monitoring of vascular leakage and edema. Vascular leakage was monitored radiometrically using $99 \mathrm{mTc}$-diethylenetriaminepentaacetic acid, which is a nondiffusible tracer for the assessment of BBB disruption, while edema was verified by histological analysis. The cumulative effects of chemical preconditioning by cobalt and hypobaric hypoxia were also studied. Genes that were common in all of the 3 groups studied (hypoxia, cobalt, cobalt +hypoxia) and those that were distinct for one particular group were also identified by microarray. Some of the genes were confirmed by reverse transcriptase-polymerase chain reaction and a few were validated at the protein level. Pathway predictions were also performed using KEGG software highlighting certain pathways affected by these treatments. These results suggest the involvement of various endogenous molecular mechanisms in the protection accorded by cobalt preconditioning against deleterious effects of hypobaric hypoxia. The study may help in identifying novel therapeutic targets for treatment of cerebral edema and possible use of cobalt as a neutraceutical.

K. Shrivastava Anju Bansal L. Ganju

G. Ilavazhagan Delhi, India

Solar Activity Influence on Rats' Resistance to Hypoxia at Different Day, Time, and Seasons

Resistance to acute hypoxia was estimated as lifetime in seconds (LT) of Wistar male rats ( $n=3030$; mass, 150-180 g). They were exposed to one minute "rise" in altitude chamber to an altitude of $11.5 \mathrm{~km}$ in three different hours of the day: (1300-1500 hours = beginning; 1600-1800 hours = middle; and 1900-2100 hours = end of day) in different seasons during 4 years. This corresponds to one solar cycle at the time of solar activity (SA) rise from minimum to maximum. Solar activity (SA) influence was assessed as LT variability - percent of LT significant differences at different SA. Similarity of LT alterations was assessed as percent of the sum of similar LT alterations of all significant LT alterations of compared seasons or day periods. The SA was estimated by daily sunspot num- bers: 0 to $49=$ low SA; 50 to $99=$ middle $\mathrm{SA}$; and 100 and greater $=$ high SA.

There were 2 to 7 stages of LT alteration at SA rise at different day periods of seasons, LT having altered 1.2 to 14.0 times. Lifetime in seconds was maximum at low SA and middle SA; LT was minimum at any SA. Lifetime in seconds diminished for the most part of all LT alterations at SA rise. Lifetime in seconds increased $7.7 \%$ to $22.7 \%$ of all LT alterations during a day, being 2.1 to 3.0 times more frequent in winter and spring than in summer and autumn. Lifetime in seconds increased equally in the middle and at the end of day in winter and in all day periods of spring; LT increased more often in the middle of the day and at the end of day in summer and at the beginning of the day in autumn. Lifetime in seconds variability during a day was $15.9 \%$ in winter, $27.7 \%$ in spring, $32.6 \%$ in summer, and $31.1 \%$ in autumn. At identical SA rise, LT variability was greater in summer and autumn than in winter and spring (1.6-2.0 times), was minimum in the winter, and grew toward autumn (in the middle and at the end of day): winter $<$ spring $<$ summer $<$ autumn. Lifetime in seconds alterations were more similar in winter and summer and in spring and autumn. Similarity of LT alterations was maximum in winter and summer (40\%). There were no similarity of LT alterations in spring and summer. Similarity of LT alterations in different seasons was greater in the middle of the day and at the end of day than at the beginning of the day (4.0-11.3 times). At similar SA rise at different season periods, LT variability was greater at the beginning of the day and in the middle of the day in summer and at the end of the day in other seasons. Similar LT alterations in all day periods were almost identical in all seasons (about 30\%-40\%). All LT alterations mentioned above were significant $\left(P<.05-10^{-5}\right)$.

Solar activity influences LT in about $16 \%$ to $30 \%$ of cases during a day for different seasons. Type of SA influence on LT depends on season and time of day in more than $60 \%$ of cases.

Marina Khachaturyan, $\mathrm{PhD}$ Moscow, Russia

\section{Models and Algorithms for Probabilistic Safety Assessment of Intermittent Hypoxia Training/Treatment}

Individual variability of physiological reactions to inhalation of hypoxic gas mixtures is substantial; hence, the importance of a hypoxic test that establishes a hypoxia reaction type and individualizes the treatment regime is evident. Intermittent hypoxia training (IHT) in various forms becomes more and more popular now in medical practice for treatment and prevention of many diseases as well as in sport training for the improvement of physical endurance.

Meanwhile, a reduction in the partial pressure of inspired oxygen may be the risk factor threatening negative events and even sudden death. During the past few years numerous debates about the competence of diagnostic and therapeutic usage of hypoxia in humans have been noted. At the same time, there is the lack of evidence related to the strong evaluation of the risk/benefit ratio. The analysis of such a ratio for hypoxic treatment application is complicated due to the differences in cri- 
teria for individual dosage and utilized methods. The special solicitudes appear when the use of hypoxic training for children is discussed. When the hypoxic tests are used for the prognosis of altitude adaptation in adults, another problem appears: moderate hypoxia does not reveal well the distinguishing individual features of adaptability, whereas tests with more severe hypoxia (which are more dangerous) allow us to determine a better prognosis. For probabilistic safety assessment of IHT application to adults and children, the method comprising the formalized description and assessment of physiological reserves is offered. The method is called the "Method of Expert Assessing Scales" (MEAS). MEAS dilates capabilities of traditional probabilistic safety assessment (PRA). MEAS models estimate the hazard at the stage of its origin (ie, during the latent period). The combination of MEAS with traditional PRA methods enables us to assess operatively for each situation the degree of potential hazard for an organism and to analyze its causes. Thus, MEAS establishes an analytical relationship between physiological parameters and estimations of basic event probabilities. Then these outcomes will be used by methods of traditional PRA for the calculation of complication probabilities. As a result, the hazard can be estimated at the earliest stage of its development. It enables us to arrange avoidances of hazard when these measures are most well-timed and effective. Thus, the complex application of logical-probabilistic methods and MEAS can become a model-algorithmic basis for information technology of the analysis of an organism's state under hypoxic loading and can forecast pathological event hazards during the continuation of the procedure. These and others problems associated with diagnostic and therapeutic usage of hypoxic impacts upon humans require further elaboration of both medical and mathematical possibilities.

Alexander Serebrovsky, PhD Tatiana Serebrovska, $\mathrm{PhD}$ Kiev, Ukraine

\section{Approaches, Equipment and Regimes for Intermittent Hypoxia Training}

Any progress in the application of scientific achievements to human practice depends on the development of concrete methodic principles and engineering innovations. The concept of repeated hypoxic training arose before the Second World War because of the need for altitude acclimatization of pilots. From that time on the intermittent hypoxia training (IHT) method underwent essential overpatching both in methodology and technical implementation. Intermittent hypoxia training has been advocated for prevention and treatment of some diseases, such as bronchial asthma, coronary heart disease, myocardial infarction, essential hypertension, Parkinson's disease, and obesity. Various mechanisms for the beneficial effects of IHT have been hypothesized. Principals of IHT utilization for cell cultures, animal experiments, and human activities were elaborated. In vitro intermittent hypoxia: To investigate mechanisms associated with the cellular effects of IHT, studies on cell culture models became popular. Research utilizing geno- mic and proteomic approaches may provide important clues to the mechanisms by which IHT leads to hypoxia-induced adaptations. Equipment for animal training: Much attention has been paid during the decades to both hypobaric and normobaric hypoxia training on animals, such as rats, mice, dogs, cats, rabbits, pigs, horses, and even insects. Different types of altitude chambers, normobaric flowing chambers, and breathing masks are widely used for such experiments. Equipment for human normobaric hypoxic training: The disadvantages of hypobaric chambers, which were widely used in the 20th century, have provoked intensive studies of normobaric hypoxia training, and in recent years, normobaric breathing of hypoxic gas mixtures has become a practical means of producing IHT. A traditional treatment protocol for IHT comprises repeated exposures to hypoxic air breathing, alternated with breathing ambient (normoxic) air. Three main methods are currently available: 1) hermetically sealed cabins for several patients, in which $\mathrm{O}_{2}$ concentration is reduced to $12 \%$ to $14 \%$; 2) individual hypoxic devices operating on the open breathing principle; and 3) administration of gradually intensifying hypoxia using a rebreathing technique with $\mathrm{CO}_{2}$ elimination. Individual reaction types: The marked individual variability both in animals and humans in the response to, and tolerance of, hypoxia is described. Estimation of human individual sensitivity to hypoxia is important for selection of individual IHT regimes during sport training or treatment of diseases. The special programs were developed to carry out hypoxic training according to individual reactivity. The optimal regime for a particular individual lies in the correct timing, duration, and severity of hypoxic exposure during 1 session and during the number of sessions.

Tatiana V. Serebrovska, PhD Kiev, Ukraine

\section{Breath-Hold Diving and Decompression Sickness}

Breath-hold (BH) diving is practiced in many parts of the world as a sport, in order to get pearls and as a record-breaking activity. The pearl divers in the South Pacific typically perform repetitive $\mathrm{BH}$ dives to depths of $30 \mathrm{~m}$ or more for 1.5 to 2.5 minutes for about 6 hours a day. Some of the divers developed what was called "Taravana," a disease similar to decompression sickness (DCS) and suspected to be due to nitrogen accumulation. The divers who developed "Taravana" primarily were having symptoms from the central nervous system, including nausea, dizziness, and partial or complete hemiplegia. Paulev argued for a connection between DCS and BH diving (1965, 1967). Paulev and 3 other divers developed symptoms of DCS after a series of BH dives in a submarine escape training tank in Norway. In each case, severe neurological symptoms and signs were present.

One of the objectives has been to develop a suitable computer model and to calculate the amount of nitrogen-expressed as nitrogen tissue tensions-accumulated in the body during diving profiles.

Symmetric accumulation and elimination of an inert gas is described by a differential equation: 


$$
d I I / d t=0.693(P a m b \cdot t-I I) T_{1 / 2}
$$

where $I I$ is the tissue pressure of the inert gas, Pamb is the ambient pressure at the actual depth, and $T_{1 / 2}$ is the half-time period of the tissue. The differential equation is solved by numerical approximation: $I I_{1}=I I_{0}+0.693(\mathrm{Pamb} \cdot t-I I) / T_{1 / 2}$.

We have developed a dive table that may protect against decompression sickness, but its validity has to be evaluated in practice. The danger is increased in mountain lake free diving, where $\mathrm{BH}$ diving has not yet been practiced but probably will be in the near future.

Hans Christian Møller Thorsen MD

Poul-Erik Paulev, MD, Dr Med Sci

Gustavo Zubieta-Calleja, MD

La Paz, Bolivia

\section{Acute Mountain Sickness-Can it be Predicted?}

Acute mountain sickness (AMS) is a complex syndrome seen in people reaching altitudes. Acute mountain sickness is frequently encountered when the gain in altitude is rapid with an improper acclimatization schedule.

Aim.-To evaluate the role of capillary fragility and serum electrolytes as predictors in AMS.

Materials and Methods. - The study was done on 3 groups. Group I consisted of a team of 12, trekking and climbing in the Ladakh Himalaya. Group 2 comprised 15 students and 5 instructors attending the mountaineering course at the Nehru Institute of Mountaineering. Group 3 had 6 climbers and 7 highlanders (porters) who were on an expedition to peak Kamet. Group 1 was evaluated at Bangalore (1000 m), Leh (3800 $\mathrm{m}$ ), and Kangri Base Camp (5000 m); Group 2 was evaluated at Uttarkashi $(800 \mathrm{~m})$, Base Camp (4000 m), and Advance Base Camp $(5000 \mathrm{~m})$ before and after attempt on peak Dudhana $(6200 \mathrm{~m})$. Group 3 was evaluated at Malari Base Camp (3133 m), Advance Base Camp (5000 m), Camp III (7130 m), and Camp IV $(7600 \mathrm{~m})$. The evaluation included a thorough clinical examination, including retinal fundoscopy and the Hess test. Estimation of sodium and potassium in sweat, saliva, and urine and symptoms and signs were assessed at each evaluation point. The test for capillary fragility was done at the first evaluation point.

Results.-The initial physical evaluation of all 3 groups was normal; based on these results, all were cleared for the expeditions. In Group 1 all 12 members had mild headache at Leh $\mathrm{G}(3800 \mathrm{~m})$, but these headaches disappeared completely with 2 to 3 days of acclimatization. All 12 reached the Base Camp at $5000 \mathrm{~m} ; 7$ had mild headaches and 2 had nausea. Six of these climbers recovered completely over the course of 2 days. In Group 2, 1 of the 15 students, an athlete of 21 years, developed headache and nausea at Advance Base Camp (5000 $\mathrm{m})$. In Group 3, on expedition to Kamet, 4 climbers and 4 highlanders tested positive for mucosal petechiometry (checked at Malari Base Camp, 3133 m). Of these, 4 developed signs and symptoms of AMS at Advance Base Camp (5000 $\mathrm{m})$ and needed evacuation to Base Camp. Two developed sim- ilar problems at Camp III (7130 m) and 2 at Camp IV (7600 $\mathrm{m})$. At each instance, being nonresponsive to medical measures, the individuals had to be evacuated to lower camps, which resulted in full recovery. Analysis of the data shows that all those who were positive for the test of capillary fragility developed AMS.

Summary.-The sodium and potassium levels have no predictive value. The test of capillary fragility is deranged or downregulated and is a sensitive AMS predictive tool.

K. Govind Babu MD

R. Srinivasa, MD

Thuppil Venkatesh, PhD

Vidyasagar Casikar, MD

Bangalore, India

\section{Expression of Endogenous Antioxidants in Rat Hippocam- pal Neurons After Four Kinds of Hypobaric Hypoxia Mod- eling High Altitude}

Hypobaric hypoxia produced in chamber is a model of high altitude. Four models of hypoxia were studied: severe ( 3 hours, 180 Torr $=11000 \mathrm{~m})$; 1 -time mild $(2$ hours, 360 Torr $=5000$ $\mathrm{m})$; 3-time mild (once in the day); and severe 1-day after 3time mild one. The severe hypoxia caused extensive neuronal damage in the hippocampus and 50\% rat mortality. Preconditioning by 3-time (but not 1-time) mild hypoxia prevented severe hypoxia-induced neuronal death and decreased animal mortality.

The effect of hypoxia on the expression of $\mathrm{Cu}, \mathrm{Zn}$-depending superoxide dismutase ( $\mathrm{Cu}, \mathrm{Zn}$-SOD), Mn-depending superoxide dismutase (Mn-SOD), thioredoxin-1 (Trx-1), and thioredoxin-2 (Trx-2) was studied in rat hippocampal fields: cornu Ammonis-1, 2 and 3 (CA1, CA2, CA3) and dentate gyrus (DG). The changes in expression were studied by immunocytochemistry.

Severe hypoxia induces expression of all antioxidants (AO) studied. The significant increase in Mn-SOD, Trx-1, and Trx2 levels was evident 3 hours following hypoxia; the increase in $\mathrm{Cu}, \mathrm{Zn}$-SOD was seen at 24 hours. The preconditioning with repetitive 3-time mild hypoxia prominently augmented this induction 3 hours after severe hypoxia in comparison with nonpreconditioned animals (except Mn-SOD in CA1 and DG). At 24 hours the difference between non-preconditioned and preconditioned rats was reduced.

At 24 hours after 3-time mild hypoxia alone the immunoreactivity of $\mathrm{Cu}, \mathrm{Zn}-\mathrm{SOD}$, Trx-1, and Trx-2 was decreased compared with controls. Expression of Mn-SOD was increased in CA1 and DG but not in CA2 and CA3.

At 24 hours after 1-time mild hypoxia the immunoreactivity of $\mathrm{Cu}, \mathrm{Zn}-\mathrm{SOD}$, Trx-1, and Trx-2 was also decreased compared with controls. Expression of Mn-SOD was increased in CA1, decreased in DG, and was not changed in CA2 and CA3.

Thus, the effects of 1- and 3-time mild hypoxia alone on $\mathrm{AO}$ expression are similar. So augmentation of AO expression after severe hypoxia in 3-time preconditioned rats and the difference between effects of 1- and 3-time preconditioning on 
cells surviving following severe hypoxia are not due to AO level at the starting point of severe hypoxia. The dynamics of AO expression in 4 time-points ( 3 hours and 24 hours after the first mild hypoxia and 3 hours and 24 hours after the third one) represents phase oscillation. Most likely this oscillation is important for the mechanism of hypoxic tolerance induced by 3 -time preconditioning.
Sergei A. Stroev

Tampere, Finland; St Petersburg, Russia Ekaterina I. Tjulkova Michail O. Samoilov St Petersburg, Russia Markku Pelto-Huikko Tampere, Finland 